\title{
Performed Procedure Step SOP Class UID
}

National Cancer Institute

\section{Source}

National Cancer Institute. Performed Procedure Step SOP Class UID. NCI Thesaurus.

Code C69262.

The unique identifier for a performed procedure step class. 